\title{
Computer Vision for Color Detection
}

\author{
Deborah T. Joy ${ }^{1}$, Gitika Kaur², Aarti Chugh ${ }^{3}$ and Shalini B. Bajaj ${ }^{4}$ \\ ${ }^{1,2}$ Student, Department of Computer Science \& Engineering, Amity University Haryana, Gurgaon, India \\ ${ }^{3}$ Assistant Professor, Department of Computer Science \& Engineering, Amity University Haryana, Gurgaon, India \\ ${ }^{4}$ Professor, Department of Computer Science \& Engineering, Amity University Haryana, Gurgaon, India \\ Correspondence should be addressed to Deborah T. Joy; deborahtjoy@gmail.com
}

Copyright (c) 2021 Made Deborah T. Joy et al. This is an open access article distributed under the Creative Commons Attribution License, which permits unrestricted use, distribution, and reproduction in any medium, provided the original work is properly cited.

\begin{abstract}
Humans see the world in colors. When it comes to the aspect of just looking, all it does is please the eyes but when it comes to questioning its make, it becomes a challenge. It is much easier to be served the values without the tedious task of finding a person who understands colors. This paper proposes the idea of teaching a computer to detect and define a color well enough to have useful applications. The detection algorithm proposed uses the advantage of the camera and fed in data to detect even the color based on RGB values. The algorithm involved calls on a function that runs loops on readjusting the distance based on a nearest match. This effortlessly helps define a color based on the RGB color space with a peaking accuracy.
\end{abstract}

KEYWORDS- Color Detection, Computer Vision, RGB Color Space, Shortest Distance Algorithm.

\section{INTRODUCTION}

Color is one of the most important characteristics of an image. Color detection is the process of detecting the name of any color. It is the fundamental step in many computer vision systems and is necessary to recognize objects; it is also used as a tool in various image editing and drawing apps [1]. When using OpenCV, color detection has many advantages for instance, it allows the detection of a specific color in a live-stream video content. Color is a feature that has been exploited to a great extent in digital image processing, since it is a powerful tool that often facilitates the classification and identification of objects, which can be discriminated based on the large number of appreciable color tones [2].

The basis of most detection techniques lies in Artificial intelligence. AI refers to the simulation of human intelligence in machines that are programmed to think like humans and mimic their actions. The term may also be applied to any machine that exhibits traits associated with a human mind such as learning and problem-solving. The ideal characteristic of artificial intelligence is its ability to rationalize and take actions that have the best chance of achieving a specific goal [3].

Talking about computer vision, it is a multidisciplinary scientific field that operates on digital images or videos to automate tasks that the human visual system can do. Computer vision is an interdisciplinary scientific field that deals with how computers can gain high-level understanding from digital images or videos [4].

From the perspective of engineering, it seeks to understand and automate tasks that the human visual system can do. Computer vision tasks include gathering, processing and analyzing the information from digital images. Computer vision is a field of artificial intelligence that trains computers to interpret and understand the visual world. Using digital images from cameras and videos and deep learning models, machines can accurately identify and classify objects [5].

The idea thus is built as an application through which one can automatically get the name of the color by clicking on them. Having a data file that contains the color name and its values the distance from each color is calculated to find the shortest one. Theis paper has as well attempted to distinguish the accuracies in the different color models as well.

\section{A. RGB Color Space}

An image is basically a matrix of pixel values. An image can be represented using many color models like grey-scale, RGB, HSV etc. RGB model is used to detect the colors in an image [6]. The RGB color space comprises within an additive color model in which red, green, and blue light are added together in various ways to reproduce a broad array of colors. RGB commonly is being used in color detection, since image data captured by camera are normally provided and stored as RGB. For example, Yang et al. [7] constructed two codebooks using RGB features and local binary pattern features for visual tracking. 

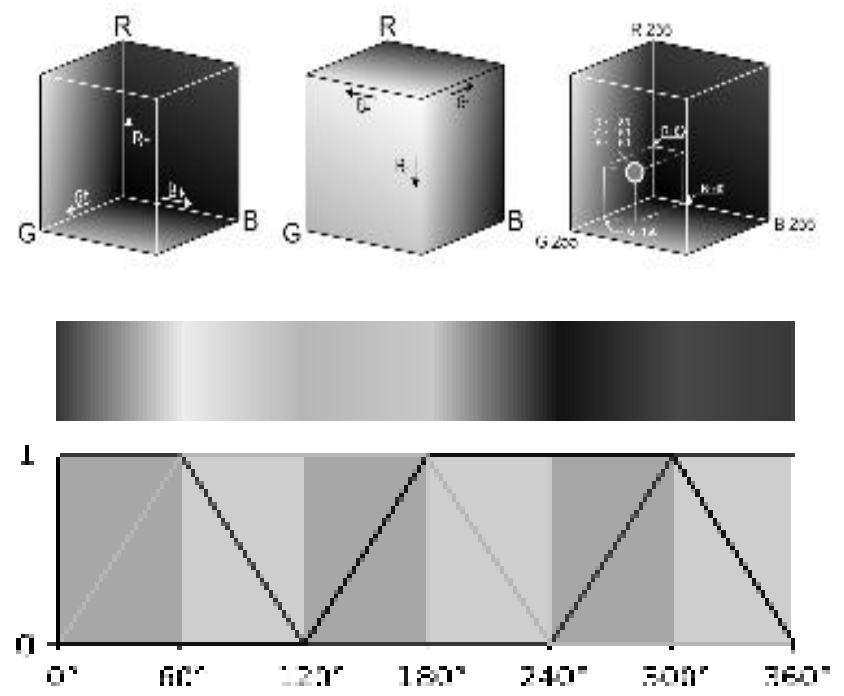

Fig. 1: RGB Color Model [4]

\section{LITERATURE REVIEW}

Colors and their vastness are brought to light in cohesion with computer vision over the years. Rubin observed that, although color is one of the most interesting and integral parts of vision, most models and methods of colorimetry available to describe and quantify color have been developed outside of optometry. His research presents a summary of some of the most popular color models and a brief history of the advancements that have led to our current understanding of the complicated phenomenon of color [8].

Behic created a basic application that helped to detect the colors in an image. The program constructed also returned the RGB values of the colors, which was really helpful. Many graphic designers and web designers' understanding on how RGB values can be helpful. Building a color recognizer is a great project to get started with Computer Vision according to them [9]. Wyszecki and Stiles proposed a research work that describes regarding the color science ideas and strategies. The RGB show is used here to acknowledge the shading within the image. The RGB show could be a shading model that joins red, inexperienced associated blue lights in numerous approaches to create an assortment of hues [10].

In their paper, Berns and Reiman discussed that image segmentation subdivides a picture into its constituent regions or objects. The amount of segmentation depends on the matter to be solved. Non-trivial image segmentation is one among the foremost tough tasks in image process. The accuracy of the segmentation determines the final word success or failure of a computerized analysis program [11]. Gonzalez, in his paper describes that MATLAB takes every answer as a network, that makes it the foremost usually used image making ready stage photos will speak to grey scale, RGB, HSV, and alternative shading models. It also goes over using MATLAB describing ways for detection of shapes and colors of objects which have been introduced antecedently [12]. Further, Abadpour and Kasaei tell relations in to conversion of RGB image to gray scale image and so to black and white (binary) image and much additional, but the study of form recognition doesn't seem to be represented. In the paper color image process using principal element analysis describes the color recognition involves comparison of every pixel within the metric and leads to the dominant color because the color of the given object is explained [13].

In their paper real time color recognition, Senthamaraikannan, Shriram and William propose new real time color recognition features, i.e., extracting primary colors for the aim of vision-based human-computer interaction. Vision-based human-computer interaction can be achieved by analyzing segmental primary color regions primarily focused on color-based image segmentation and vision primarily based color recognition by addressing these difficulties [14].

However, cluttered backgrounds, unknown lighting conditions and multiple moving objects create this task difficult. Consequent to all of this, Bhanot further led the walk to a completely different direction. Images in Python had been an area of interest for this author. Coming across OpenCV which allows import and manipulation of images in Python, the author started to wonder if information could be extracted out of those images using Machine Learning and used in some way. It has been seen that searching can be done online on the basis of certain filters one of which is color. That inspired the author to actually write the code that can extract colors out of images and filter the images based on those colors [15].

Further, in their article, Ray and Rose, explain how the basics of OpenCV are understood, how colors are extracted from images using KMeans algorithm and how images are filtered from a collection of images based on RGB values of colors. This opens the doors for many superior applications such as searching for colors in a Search Engine, or looking for a piece of clothing that has a certain color in it [16].

Color detection is a field that has many buyers. There is a plethora of methods to detect colors, ranging from physical methods to latest machine learning and even web scrapping methods. Coming over to a choice in color space, some of the prominent methods have been mentioned below thus we have the major color spaces in the assistance of color detection. One of them has been stated earlier.

\section{A. HSL Color Space}

In HSL color space, $\mathrm{H}, \mathrm{S}$ and $\mathrm{L}$ represent three different 
dimensions respectively, and each dimension comprises two threshold values (maximum and minimum values). ' $\mathrm{H}$ ' is hue which means the color name, such as yellow, red, and the range lies in $0^{\circ}$ to $360^{\circ}$, ' $\mathrm{S}$ ' is saturation which means the color of purity, the range is 0 to 100 , ' $\mathrm{L}$ ' represents the lightness and the range is 0,100 [17].

HSL has less to with the actual color definitions only more about their intensity and weight.

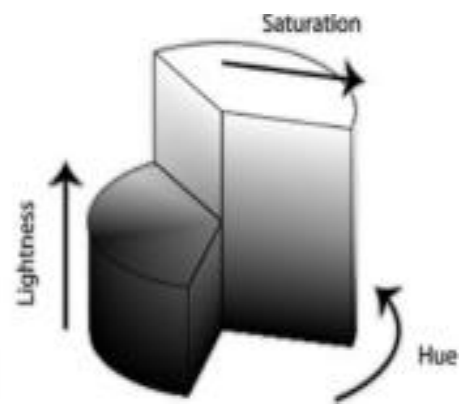

Fig. 2: HSL Color Model

\section{B. CMYK Color Space}

A CMY color space uses cyan, magenta, and yellow (CMY) as its primary colors. The CMYK color space is a variation on the CMY model. It adds black (Cyan, Magenta, Yellow, and black (Key)). The CMYK color space closes the gap between theory and practice. In theory, the extra black component is not needed. However, experience with various types of inks and papers has shown that when equal components of cyan, magenta, and yellow inks are mixed, the result is usually a dark brown, not black. Adding black ink to the mix solves this problem [18]. As compared to the additive RGB color space, the CMYK color space is subtractive in nature.
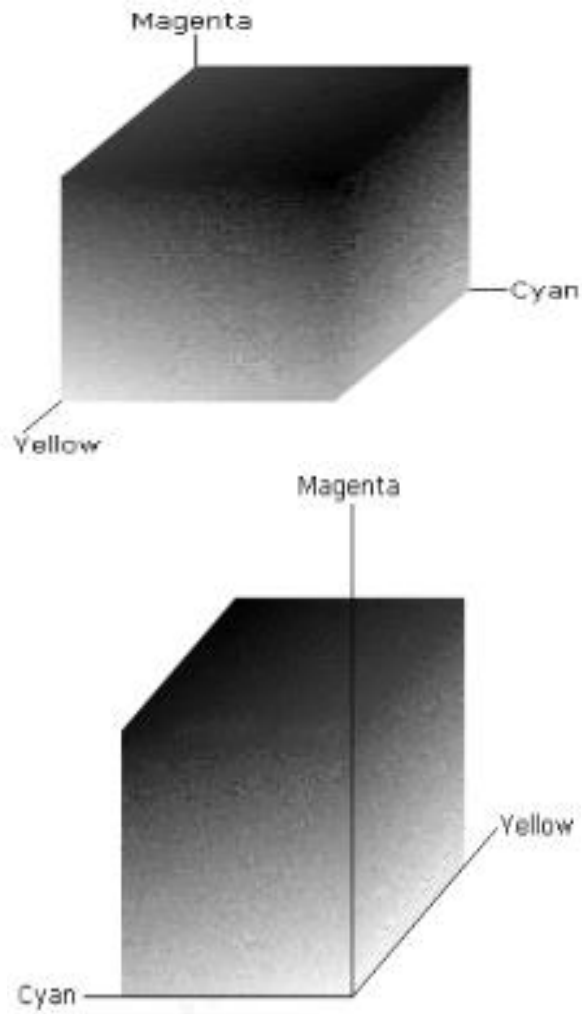

Fig. 3: CMYK Color Model

\section{METHODS ADOPTED}

After a whole lot of contemplation, the aforementioned leads the authors to believe that color detection is not restricted to just the human eyes and further has several partitions and techniques in machine interpretation of colors. Now to teach an algorithm is not hard, the implementation follows having a data file that contains the color name and its RGB values, the distance from each color is calculated to find the shortest one, it is readjusted in each iteration. So, the process goes as follows, collection of the dataset and consequently cleaning and importing it. The real magic comes here in building the shortest distance algorithm. In the given pseudo code, functions and keys are in bold and italic, mathematical calculations are highlighted in italic within quotes and variable are in bold.

1. create a function to calculate minimum distance from all colors and get the most matching color

2. set a minimum value to correspond with distance

3. while values in the file exist:

a. set distance as the "sum of absolute values of difference between image and file values"

b. reset minimum value

c. get the name of color corresponding to the new minimum

4. map "accuracy" based on value in the file and value in the image

5. plot distance loops

6. return the name of final closest valued color

In this color detection algorithm, an application is built through which you can automatically get the name of the color by clicking on them. The benefit of the said model is that the distances are calculated by successive update of the minimum distance. 


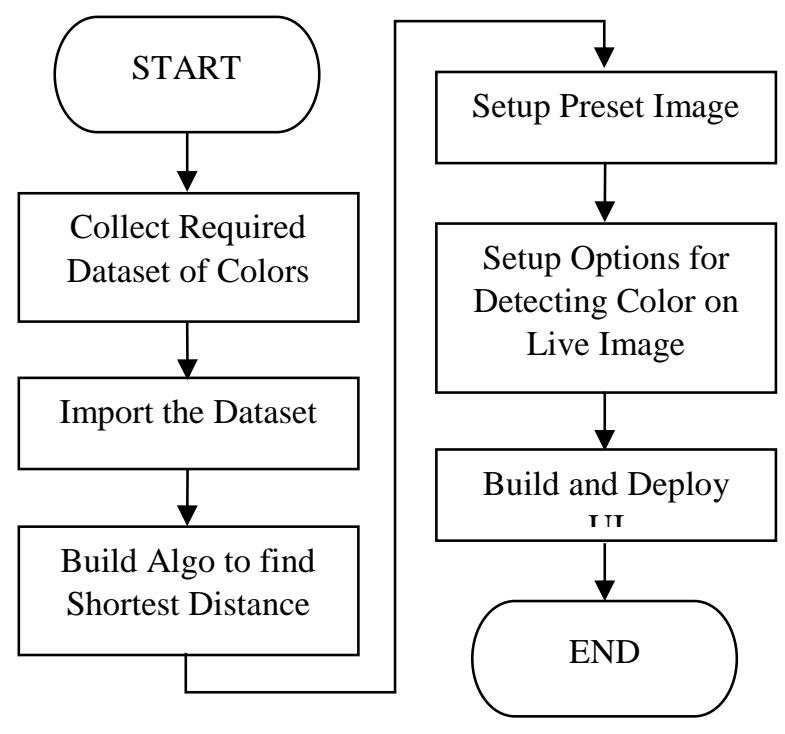

Fig. 4: Process Flow Diagram

To better understand how the idea is actually flowing above a process flow diagram has been constructed to give an illustration of how things are built. Now, Color Detection is a fairly simple process when using RGB combinations of colors as compared to CMYK and HSL methods. RGB gives a comparatively better accuracy. So the accuracy is calculated by mapping the original RGB values and finding its error with the actual color values. This is further followed by the user interface setup. A default image, as discussed later, is setup to require a color definition further a GUI is setup using python as base language to let a user click a fresh image and find its RGB value along with the color match closest to it. Here it is useful to understand that the libraries offered by the languages help with most of the work. Computer vision has its own library OpenCV to make the analysis of images a much easier task. Python is a high level, interpreted, interactive and object-oriented language which is also highly readable. While the OpenCV library has more than 2500 algorithms and is capable of processing images and videos to detect faces, identify objects, classify human actions, track moving objects, color detection, pattern recognition and many more [19].

An activity diagram portraying the control flow from a start point to a finish point and as well showing the various decision paths that exist while the activity is being executed has been given below.

An activity diagram is a behavioral diagram i.e. it depicts the behavior of a system. Both sequential processing and concurrent processing of activities have been depicted using the activity diagram.

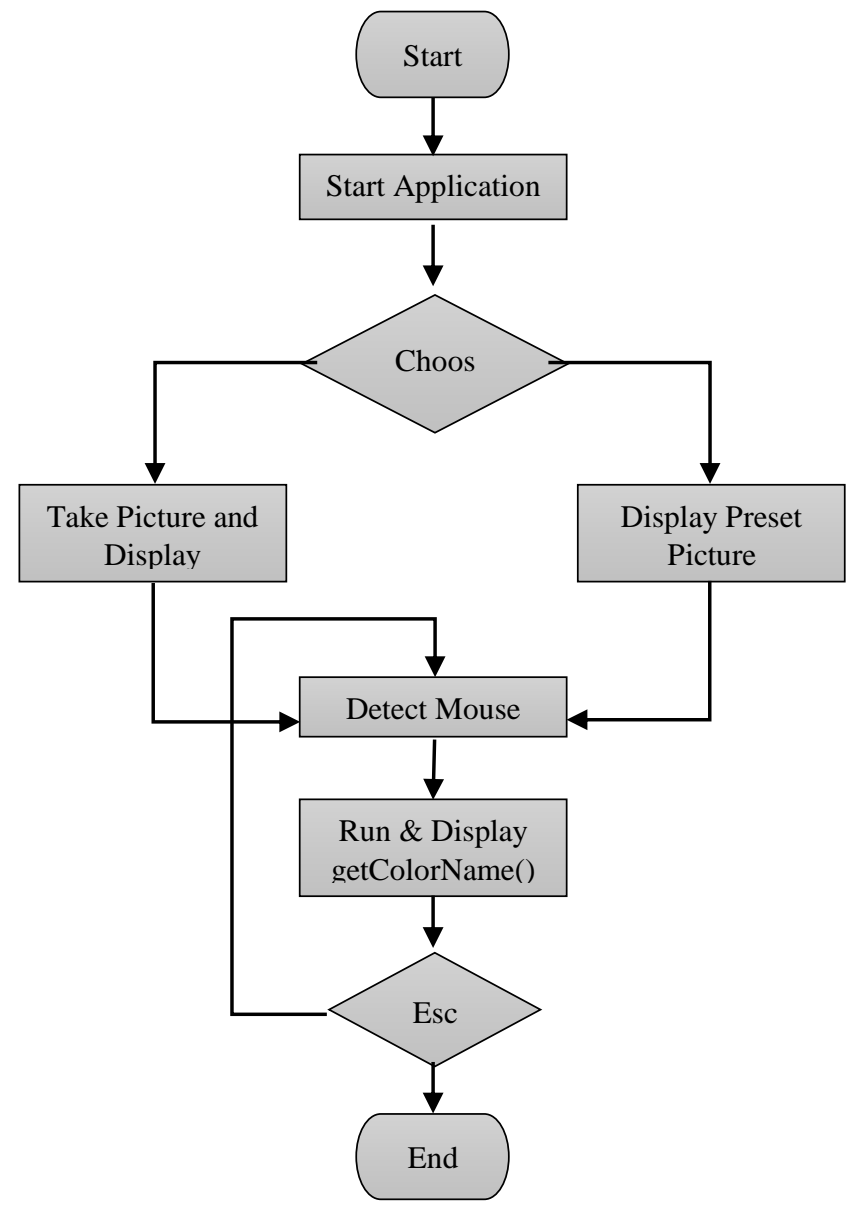

Fig 5: Activity Diagram for System

\section{EXPERIMENTAL RESULTS}

The RGB color model giving the accuracy that it is leads to believe that the other color models as well may have a wing of accuracy. Further research has revealed a neat difference in the color models, which have been discussed below. 

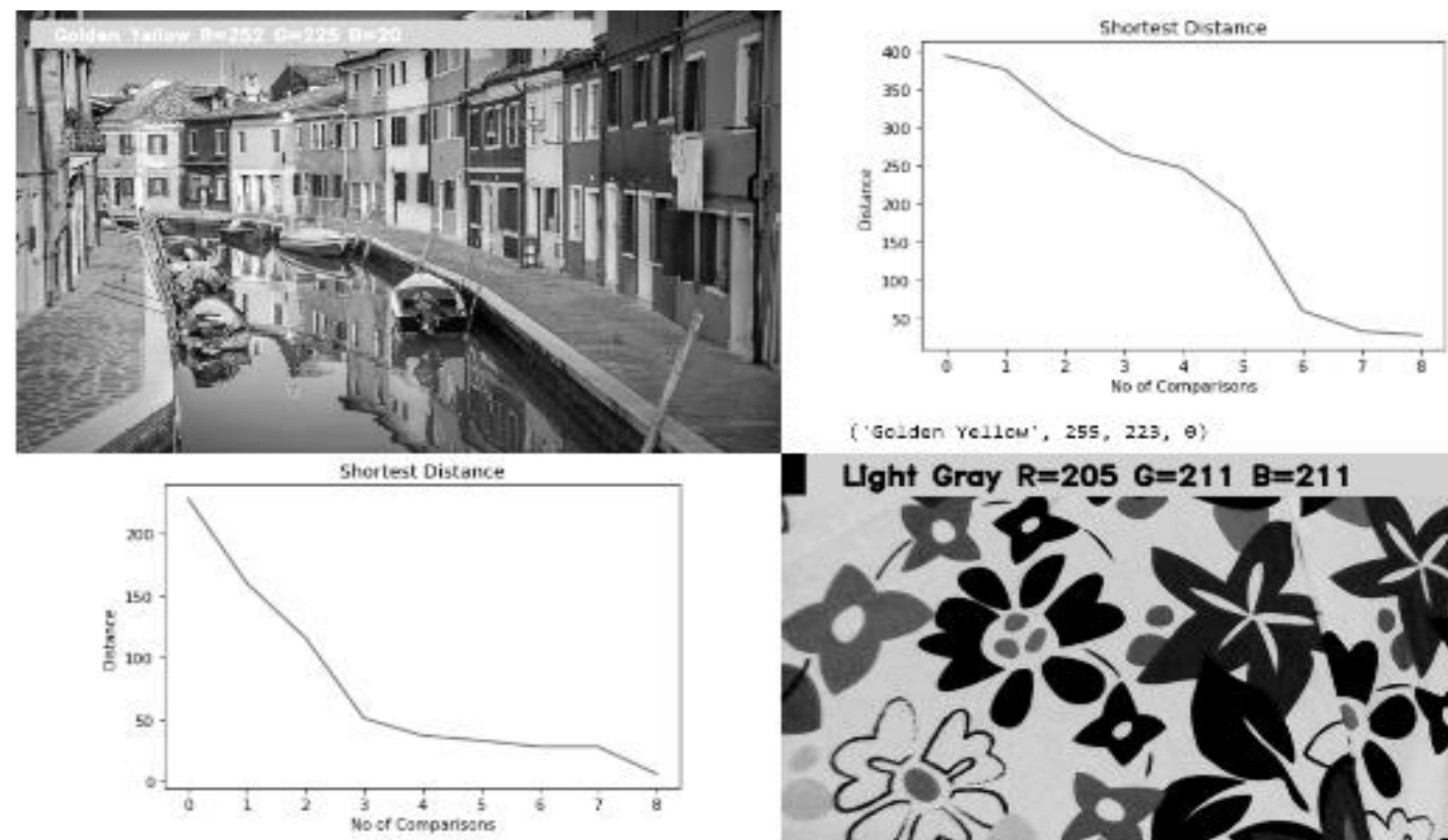

('Lighe Gray', 211, 211, 211)

('Godden rellow', 255, 223, e)

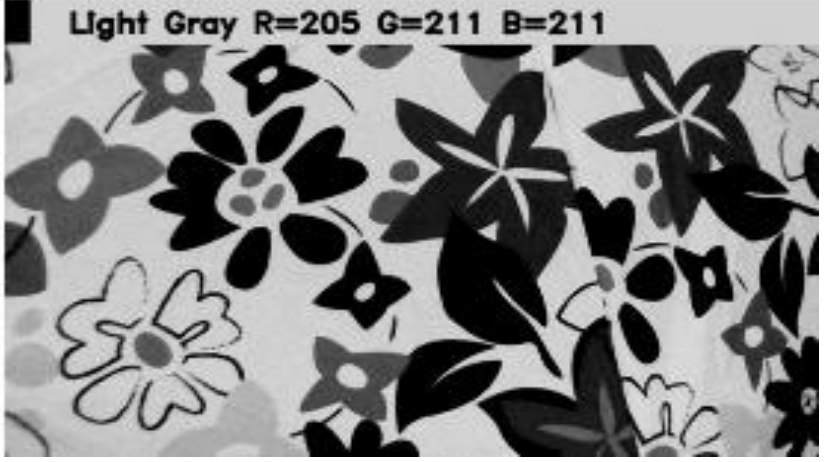

Fig. 6: Distance Calculation Comparison

The figure above offers a stark comparison on why an HD picture gives better results as defiant to picture taken by a dependent hardware, webcam in this case. What human eye would call white, in a picture leads to light grey. The graphs depict a closure in the distances after successive update to adjust the minimum distance value in each loop. Further talking about the accuracy, in the case of RGB distance calculation algorithm, the accuracy can be improved by changing the updated distances. The added advantage of RGB model is classification on neutral all color grounds, making it easy to calculate distances repeatedly with more accuracy as compared to parallel color models. Though RGB offers some debatable disadvantages and questionable advantages, if the dealing itself is complex fitting it into a computer aided system may not be advisable. All work on how color is viewed by a computer and given the fact that today's world is filled with their desired data, none of the color models can be shunned. We can only depend on accuracy of actual color / data viewed by human eyes since the constraints of computer vision lie in the pixel quality of the hardware it is subject to. A comparison between them has been given below.

Table 1: Comparison Between Color Models

\begin{tabular}{|c|c|c|c|}
\hline Model & Parameters & Advantages & Disadvantage \\
\hline RGB & $\begin{array}{l}\text { Red, } \\
\text { Green, } \\
\text { Blue. }\end{array}$ & $\begin{array}{l}\text { (1) No } \\
\text { transformations } \\
\text { are required in } \\
\text { displaying } \\
\text { information on the } \\
\text { screen. }\end{array}$ & $\begin{array}{l}\text { (1) Useless for } \\
\text { objects } \\
\text { specification. } \\
\text { (2) Difficult to } \\
\text { determine } \\
\text { specific color in }\end{array}$ \\
\hline
\end{tabular}

\begin{tabular}{|c|c|c|c|}
\hline & & $\begin{array}{l}\text { (2) Additive } \\
\text { property. } \\
\text { (3) Proves to be a } \\
\text { computationally } \\
\text { practical system. } \\
\text { (4) Ease in AI } \\
\text { applications. }\end{array}$ & $\begin{array}{l}\text { RGB model. } \\
\text { (3) RGB reflects } \\
\text { the use of CRTs. }\end{array}$ \\
\hline CMY(K) & $\begin{array}{l}\text { Cyan, } \\
\text { Magenta, } \\
\text { yellow, } \\
\text { and Black }\end{array}$ & $\begin{array}{l}\text { (1) Commonly } \\
\text { used for } \\
\text { production of } \\
\text { printed color. }\end{array}$ & $\begin{array}{l}\text { (1) Since it is a } \\
\text { subtractive } \\
\text { model, the } \\
\text { components are } \\
\text { pigments or inks, } \\
\text { not colors. }\end{array}$ \\
\hline $\begin{array}{l}\text { HSL/ } \\
\text { HSI }\end{array}$ & $\begin{array}{l}\text { Hue, } \\
\text { Saturation, } \\
\text { Lightness/ } \\
\text { Hue, } \\
\text { Saturation, } \\
\text { Intensity }\end{array}$ & $\begin{array}{l}\text { (1) Preferable for } \\
\text { users view since } \\
\text { the components } \\
\text { are correlated } \\
\text { better with human } \\
\text { perception of } \\
\text { color. } \\
\text { (2) The } \\
\text { chrominance } \\
\text { components (H } \\
\text { and S) are } \\
\text { associated with } \\
\text { the way humans } \\
\text { perceive. }\end{array}$ & $\begin{array}{l}\text { (1) Undefined } \\
\text { achromatic hue } \\
\text { points are } \\
\text { sensitive to value } \\
\text { deviations of } \\
\text { RGB and } \\
\text { instability of hue, } \\
\text { due to the } \\
\text { angular nature. } \\
\text { (2) Does not } \\
\text { supply with } \\
\text { insight for color } \\
\text { manipulation. } \\
\text { (3) Not uniform. }\end{array}$ \\
\hline
\end{tabular}


This must now give a clear idea of why what has been done in this paper has actually been done. To give an even concise comprehension the chart below, enunciates the comparison of the accuracy and precision. The green bar is for accuracy, and the blue for precision. In case of RGB the accuracy has been found a peaking 97 while CMYK gives 79 [19] and HSL offers 90 [20]. The precisions are 99, 76 and 93 respectively.

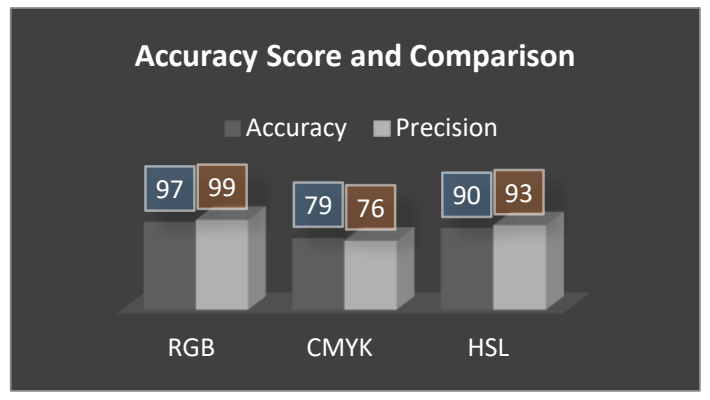

Fig. 7: Accuracy Score of Color Models

\section{CONCLUSION AND FUTURE WORK}

The Color Detection done using computer vision has been a achieved. The GUI has been successful in giving the users an option to choose from thus returning the correct RGB values when the picture is double clicked over a certain color. Using the algorithm of mapping RGB values by finding the shortest distance and managed mean in the three, the accuracy calculated by the error in RGB distance has hit $97 \%$. Color Detection in real life is a blooming concept. But there is still much to uncover. With changing technology in a fast paced and impatient world, we can look forward to heights that now seem unattainable. For instance, the interest of fashion designers or graphic designers in detecting and using certain colors that they are unable to name or find. The future scope of this project ranges from imbuing AI with being able to classify whether the colors detected are completely formal or do the hold the parameters of a device constraining the very aspect of accuracy that must be achieved and thus the color detection might in fact be faulty

\section{CONFLICTS OF INTEREST}

The authors declare that they have no conflicts of interest.

\section{REFERENCES}

[1] Gonzalez, R.C., Woods, R.E.: Digital Image Processing. Pearson (2018)

[2] Project in Python - Color Detection using Pandas \& OpenCV. https://data-flair.training/blogs/project-in-pythoncolor-detection/

[3] Jake Frankenfield, Gordon Scott. Artificial Intelligence (AI). https://www.investopedia.com/terms/a/artificial-intelligenceai.asp\#: :text=Artificial\%20intelligence\%20(AI)\%20refers\% 20to,as\%20learning\%20and\%20problem\%2Dsolving.

[4] Victor Wiley, Thomas Lucas. Computer Vision and Image Processing: A Paper Review. International Journal Of Artificial Intelegence Research. ISSN: 2579-7298, Vol 2, No 1, June 2018, pp. 28-36 DOI: 10.29099/ijair.v2i1.42

[5] Computer Vision. What it is and why it matters. https://www.sas.com/en_in/insights/analytics/computervision.html
[6] R. S. Berns, "Principles of Color Technology" (3rd edition New York: Wiley, 2000)

[7] Yang, F., Lu, H., Zhang, W., Yang, G.: 'Visual tracking via bag of features', IET Image Process., 2012, 6, pp. 115-128 (doi: 10.1049/iet-ipr.2010.0127)

[8] Hasting, G. \& Rubin, Alan. (2012). Color spaces - a review of historic and modern color models*. African Vision and Eye Health. 71. 10.4102/aveh.v71i3.76.

[9] Behic Guven. Building a Color Recognizer in Python. Towards Data Science. https://towardsdatascience.com/building-a-color-recognizerin-python-4783dfc 72456

[10] G. Wyszecki and W. S. Styles, "Color Science: Concepts and Methods, Quantitative Data and Formulae" (2nd edition New York: Wiley, 1982)

[11] Berns, RS \& Reiman DM, "Color managing the third edition of Billmeyer and Saltzman's Principles of Color Technology", Color Research \& Application, Vol.27, No.5, (2002), pp.360-373.

[12] Gonzalez RC, Woods RE \& Eddins S.L, Digital Image Processing Using MATLAB, Pearson Education, Inc, (2004).

[13] Arash Abadpour and Shohreh Kasaei. Principal Color and Its Application to Color Image Segmentation. http://abadpour.com/files/html/178si/W178si.html

[14] Senthamaraikannan D, Shriram S \& William J, "Real time color recognition", International Journal of Innovative Research In Electrical,Electronics, Instrumentation And Control Engineering, Vol.2, No.3,(2014).

[15] Karan Bhanot. Color Identification in Images. Towards Data Science. https://towardsdatascience.com/color-identificationin-images-machine-learning-application-b26e770c4c71

[16] Ray Siddheswar, and Rose H. Turi. "Determination of number of clusters in k-means clustering and application in color image segmentation." In Proceedings of the 4th international conference on advances in pattern recognition and digital techniques, pp. 137-143. 1999.

[17] Shun-Hung Tsai, Yu-Hsiang Tseng, A novel color detection method based on HSL color space for robotic soccer competition, Computers \& Mathematics with Applications, Volume 64, Issue 5, 2012, Pages 1291-1300, ISSN 08981221, https://doi.org/10.1016/j.camwa.2012.03.073.

[18] John Kennedy, Steven White. CMY and CMYK Color Spaces. https://docs.microsoft.com/enus/windows/win32/wcs/cmy-and-cmyk-color-spaces

[19] OpenCV Python Tutorial - Implementation of Computer Vision https://data-flair.training/blogs/opencv-pythontutorial/

[20] Resti, Yulia \& Burlian, Firmansyah \& Yani, Irsyadi \& Rosiliani, Dinda. (2020). Analysis of a cans waste classification system based on the CMYK color model using different metric distances on the k-means method. Journal of Physics: Conference Series. 1500. 012010. 10.1088/17426596/1500/1/012010.

[21] Cheng-Jin Du, Da-Wen Sun, 18 - Quality Evaluation of Pizzas, Editor(s): Da-Wen Sun, In Food Science and Technology, Computer Vision Technology for Food Quality Evaluation, Academic Press, 2008, Pages 427-446, ISBN 9780123736420, https://doi.org/10.1016/B978-0123736420.50021-1. 


\section{ABOUT THE AUTHORS}

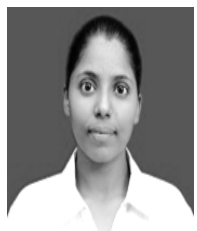

Ms. Deborah T. Joy has a Bachelor of Technology in Computer Science from Amity University. She has worked with Machine Learning, Deep Learning, Computer Vision, Sentiment Analysis and the like over the course of her education. She has published papers with national conferences, she as well has a book chapter to her credit. She has four projects and five certifications in various areas of computer studies. Her fields of interest are Computer Vision, ML/DL and AI in Healthcare.

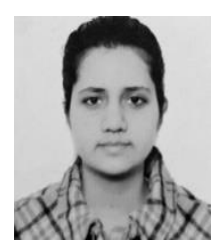

Ms. Gitika kaur has a Bachelors in Computer Science from Amity university. With a minor Degree in Artificial Intelligence she has undergone internships with Translab Technologies, Amity University and IIT Jammu. Apart from her internships and bachelor's, She pursued many courses regarding her field and interests. She underwent multiple courses to enhance her skills and complete various courses such as Python, $\mathrm{R}$ programming , Data analysis with Python, Specialization course in Statistics with Python and so on from both offline institute and online education platforms. She has also developed five varied project and presented paper in national conferences. Her areas of interest are Artificial Intelligence, Data Science and Data analytics.

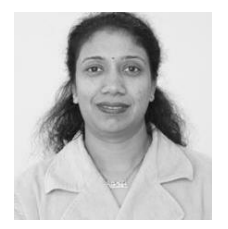

Ms. Aarti Chugh is currently an assistant professor at Amity University Haryana, India. She is research scholar at Jagannath University, Jaipur, India. She has published 15 research articles in wellknown journals and presented her research in various conferences, and attended several national and international conferences and workshops. Her research interests include Big Data, Database Systems, Image Processing, Software Testing, and Computational Intelligence

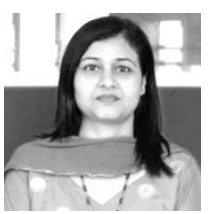

Dr. Shalini Bhaskar Bajaj did her Bachelor of Engineering in Computer Science and Engineering from Deenbandhu Chhotu Ram University, Haryana. After completing her graduation, she worked with Liberty Footwear as System Analyst for a period of six months. Thereafter, she worked as System Analyst in Appware Technologies, LLC, Dubai. Before joining GD Goenka University she worked with ITM University, Gurgaon. She completed her Master of Engineering in Computer Technology and Applications from Delhi Technological University and Ph.D. from Indian Institute of Technology Delhi (IITD), New Delhi. She has to her credit twentytwo years work experience on different projects, teaching and research in the field of Data mining. She has published more than fifty research papers in different national and international journals and presented paper in conferences. Her research interests include Data mining and Databases. 